\title{
W-band Sheet Beam Klystron Design
}

\author{
Stanford Linear Accelerator Center \\ 2575 Sand Hill Road, MS 33 \\ Menlo Park, CA 94025
}

G. Scheitrum, G. Caryotakis, A. Burke, A. Jensen, E. Jongewaard,

A. Krasnykh, M. Neubauer, R. Phillips, K. Rauenbuehler

USA

glenn@slac.stanford.edu

\begin{abstract}
Sheet beam devices provide important advantages for very high power, narrow bandwidth RF sources like accelerator klystrons [1]. Reduced current density and increased surface area result in increased power capability, reduced magnetic fields for focusing and reduced cathode loading. These advantages are offset by increased complexity, beam formation and transport issues and potential for mode competition in the ovennoded cavities and drift tube. This paper will describe the design issues encountered in developing a $100 \mathrm{~kW}$ peak and 2 $\mathrm{kW}$ average power sheet beam k1ystron at W-band including beam formation, beam transport, circuit design, circuit fabrication and mode competition.
\end{abstract}

\section{Introduction}

Sheet beam devices are particularly suited for narrow band, high peak and average power applications. The narrow bandwidth is a consequence of the low cavity impedance (R/Q) of the wide, overmoded cavities employed. In order to get reasonable impedance, the $\mathrm{R} / \mathrm{Q}$ of the cavity must be multiplied by a fairly high $\mathrm{Q}$, leading to a narrow bandwidth.

The increased surface area of the circuit reduces the thermal loading of the output circuit. This is very beneficial in millimeter wave sources where the reduced skin depth leads to higher circuit losses. The low beam current density reduces the required magnetic field. The planar circuit features lend themselves to lithographic or $\mathrm{CNC}$ machining processes.

\section{Beam Formation-Sbeet Beam Gun Design}

The main difficulty with the design of a sheet beam gun is the need to control the fields at the edges of the beam in both horizontal and vertical directions. The cathode and focus electrode geometry can be seen in Fig. 1. The cathode width defines the wide dimension of the beam. The cylindrical surface produces beam compression in the vertical dimension to define the narrow dimension of the beam. Since the beam is compressed in only one dimension, nominal values for area compression are limited to less than 20 (equivalent to a $400: 1$ area compression in a cylindrical beam). A focus electrode surface at the Pierce angle will provide beam edge confinement along the top and bottom edges of the beam but the shape of the focus along the curved edge of the cathode must be detennined with an accurate 3D electron trajectory code such as OmniTrak or Michelle. At the comer of the cathode, electron emission will be suppressed because both the horizontal and vertical surfaces of the focus electrode affect the field in that area.

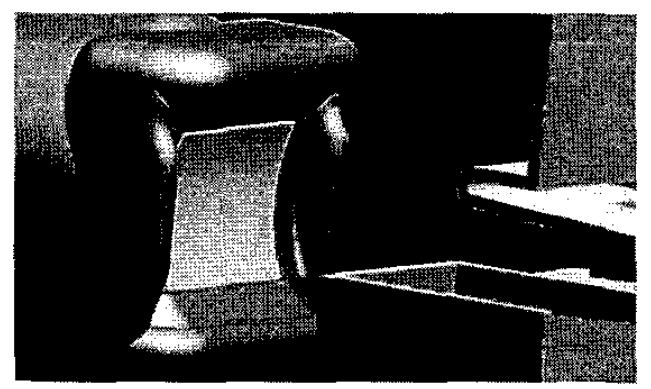

Fig. 1: Complex focus electrode shape required to control beam edges in both vertical and horizontal plane.

\section{Beam Transport}

Both periodic cusp magnet (PCM) and wiggler focusing are being considered for beam transport for the $74 \mathrm{kV} 3.6 \mathrm{~A}$ beam. PCM is favored over wiggler focusing because the $1.2 \mathrm{em}$ magnet period results in a fairly large wiggle motion that would require a wider beam tunnel. The PCM design follows the analysis ofBooske [2] and Basten [3] using offset magnets to provide focusing in the wide dimension of the beam. The magnetic field required varies from $\mathrm{I} \mathrm{kG}$ at the input to $1.5 \mathrm{kG}$ for the fully bunched beam in the output cavity. This is roughly one third of the field required for the $110 \mathrm{kV}, 2.4 \mathrm{~A}$ beam used in the pencil-beam, $95 \mathrm{GHz}$, Klystrino design.

\section{Klystron Cavity Design}

For maximum efficiency all the electrons in the sheet beam should see the same electric field across the width of the resonator. In order to produce the uniform electric field, a cutoff waveguide section is used. This produces a constant phase across the width of the drift tube. Two quarter wave matching sections are used to provide an open circuit at either end of the cutoff waveguide. Figure 2 shows a $95 \mathrm{GHz}, 3 \cdot$ gap cold test structure that would be used as a gain cavity in the sheet beam klystron.

One of the major difficulties with resonant cavities for sheet beam devices is the low cavity impedance that can be achieved. A standard cavity for a round beam klystron has an R/Q $>100$ while the RJQ for an equivalent sheet beam cavity is around 25 . To compensate for the low $\mathrm{R} / \mathrm{Q}$, multiple gaps are used in the the intermediate and output cavities. In the present design. the intermediate cavities use three gaps and the output cavity uses seven. Figure 3 shows the Ez fields for the seven gap output cavity. The number of gaps in the output cavity is not driven by bandwidth issues, rather it is necessary to achieve a reasonable 
circuit efficiency. Circuit efficiency T]ckt $=\mathrm{Qof}(\mathrm{Qe}+\mathrm{Qb})$ depends upon the relative values for intrinsic $\mathrm{Q}$ and external $\mathrm{Q}$. In millimeter wave devices, skin depth $\left(00 \cdots: \cdot 1 / \mathbf{f}^{12}\right)$ lowers $Q_{0}$ dramatically and since the cavity impedance $\mathrm{R}=\mathrm{R} / \mathrm{Q} * \mathrm{Qe}$ must be equal to the beam impedance ( $\operatorname{Vofl}_{0}$ ), a drop in $\mathrm{R} / \mathrm{Q}$ requires a higher Qe and therefore lowers circuit efficiency. Increasing the number of gaps adds to the total R/Q of the cavity and lowers the $0 \mathrm{e}$ required. The current 7 gap output cavity design has an external $\mathrm{Q}$ of approximately 500 which yields a circuit efficiency of $75 \%$.

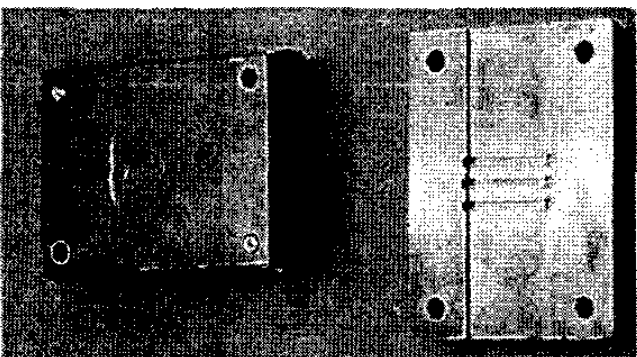

Fig. 2: Photograph of 3-gap cavity cold test assembly. The image on the right shows one half of the cold test structure with the 8 nun wide drift tube and the three resonators that operate in the $21 \mathrm{t}$ mode.

Stability calculations have been performed on the 3-gap intermediate cavities and on the 7-gap output cavity to insure that the beam current is below the start oscillation level for monotron oscillations. The analysis looks at the beam loading, Q, for the operating mode and nearby competing modes over a range of voltages up to the operating voltage. Figure 3 is a plot of $\mathrm{Qb}$ and $\mathrm{Qr}$ in the output cavity for a mode adjacent to the operating frequency. Since $\mathrm{Qr}>0$ at any voltage, the cavity is stable for this mode.

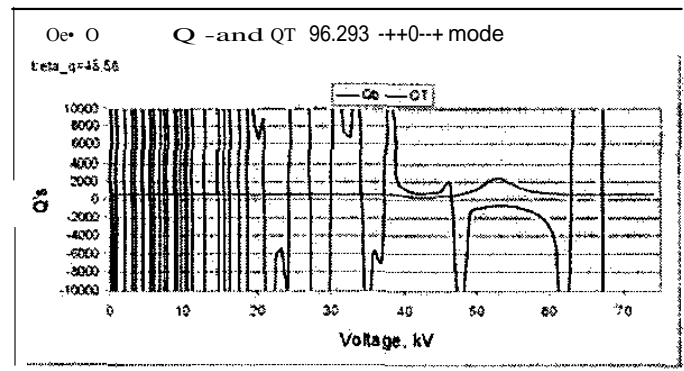

Fig. 3: Stability plot of $Q$, and $\mathrm{Qr}$ vs voltage in the 7-gap output cavity for a mode adjacent to the operating mode.

In multigap output cavities using non-relativistic beams, it is necessary to adjust the spacing between gaps to match the reduced spacing between bunches as they transfer energy to the output cavity. Fig. 4 shows the spatial tapering used to keep the bunches centered in the gaps.

\section{Circuit Fabrication}

In the previous $95 \mathrm{GHz}$ Klystrino pencil-beam design LIGA, a lithographic fabrication method, was used to make the cavities in order to maximize $\mathrm{Q}_{0}$. While the surface finish is excellent using LIGA, the turnaround time on prototype parts is quite long. In the sheet beam klystron, SLAC is evaluating normal machining of the drift tube and cavity geometry using a high speed spindle to improve surface finish.

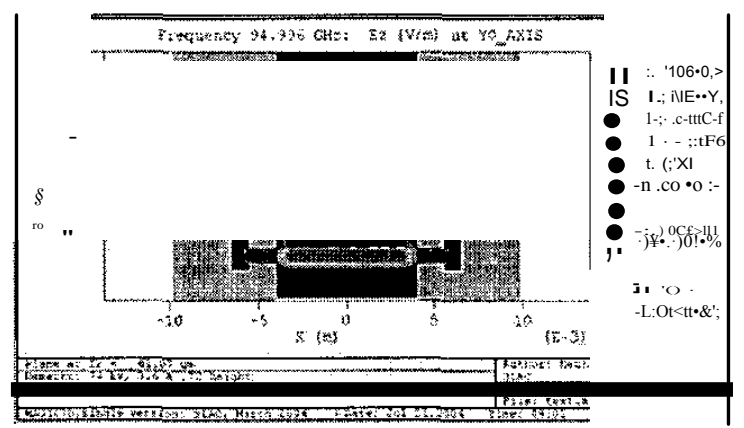

Fig. 4: Electric field plot from MAGIC 3D model of 7-gap, spatially tapered, output cavity for W-band SBK. Note field uniformity across width of beam tunnel and variation in gap spacing to match beam velocity.

Measurements will be made on the cold test parts shown in Fig. 2 both before and after a light etch and hydrogen firing. If the $\mathrm{Q}_{0}$ is within $15 \%$ of the calculated value, normal machining will be used to fabricate the circuit. The calculated intrinsic $\mathrm{Q}$ at $95 \mathrm{GHz}$ is 1616 . Initial surface finish measurement at the base of the slot is $<8$ in (<200 run).

\section{Mode Competition}

One of the major issues with sheet beam devices is that both the cavities and the drift tube are overmoded. The TM modes are cut off and their relatively wide spacing reduces the likelihood of coupling to the operating mode. The TE modes can propagate in the drift tube. In a symmetric structure with a symmetrically located beam, TE modes are not excited. Multiple MAGIC3D simulations have been run with the electron beam positioned off center in the drift tube and no evidence of TE mode generation or propagation was detected.

\section{Summary}

The design issues involved in the development of a $100 \mathrm{~kW}$, $95 \mathrm{GHz}$ sheet beam klystron are complex but tractable. The finished device is expected to weigh less than $4 \mathrm{~kg}$ in a $30 \mathrm{em} \mathrm{x}$ : $6 \mathrm{em}$ x $6 \mathrm{em}$ package.

\section{Acknowledgements}

This work is supported by the Department of Energy under Contract DE-AC03-76SF00515.

\section{References}

[I] G. Caryotakis, A. Krasnykh, M. Neubauer, R. Phillips, G. Scheitrum, D. Sprehn, R. Steele, A Jensen, D. Smithe, "Design of a $11.4 \mathrm{GHz}, 150-\mathrm{MW}$, Sheet Beam, PPMFocused Klystron' 6 th Workshop on High Energy Density and High Power RF, Berkeley Springs. WV. 2003, 22-33.

[2] J. Booske, B. McVey, T. Antonsen "Stability and confinement of nonrelativistic sheet electron beams with periodic cusped magnetic focusing", J. AppL Phys., VoL 73 No.9, May 1993,4140-4155.

[3] M. Basten, J. Booske, «Two-plane focusing of high-spacecharge sheet electron beams using periodically cusped magnetic fields)), J. Appl. Phys., Vol. 85, No. 9, May $1999,6313-6322$. 